\title{
Aus-, Fort- und Weiterbildung von Fachpersonal im Operationsdienst
}

\author{
R. Huttelmeier
}

\section{Zusammenfassung}

Im Rahmen dieses Beitrags möchte ich die vielseitigen Möglichkeiten der Aus-, Fort- und Weiterbildung für das Krankenpflegepersonal im Operationsdienst aufzeigen. Ich werde speziell auf das Berufsbild der/des Operationstechnischen Asisstentin/Assistenten $(=\mathrm{OTA})$ eingehen und durch einige kritische Anmerkungen zum Nachdenken anregen. Außerdem möchte ich auf die Bedeutung von gezieltem Personalmanagement im Operationsdienst eingehen unter Einbeziehung der Vermittlung von Schlüsselqualifikationen in der Fachweiterbildung für den Operationsdienst. Einen größeren Abschnitt meines
Artikels nimmt eine von mir befürwortete Neukonzeption der Weiterbildung für Krankenpflegepersonal in den Funktionsdiensten ein. Abschließend möchte ich über qualitätssichernde Maßnahmen im Bildungsmanagement berichten, wobei ich die Durchführung eines Pflegeforschungsprojektes als ideale Methode zur Vermittlung des Pflegeforschungsprozesses und als hervorragendes pädagogisches Instrument zur Vermittlung von Schlüsselqualifikationen favorisiere. Hinweis: Um den Lesefluss zu begünstigen, verwende ich im Text die weibliche Form. Selbstverständlich ist auch immer das männliche Geschlecht angesprochen.

\section{Einleitung}

In Zeiten des Abbaus von Planbetten in den Krankenhäusern sowie der Schließung oder Umfunktionierung ganzer Kliniken und dem damit oft verbundenen Stellenabbau ist es in der Zwischenzeit für das Krankenpflegepersonal von großer Bedeutung, durch entsprechende Fort- und Weiterbildungsmaßnahmen weitere Zusatzqualifikationen zu erlangen und dadurch als Arbeitnehmerinnen für den Arbeitsmarkt attraktiv zu bleiben. Obwohl mancherorts qualifiziertes Operationspersonal nach wie vor händeringend gesucht wird, ist es auch im Operationsdienst nicht mehr ganz so einfach, einen Arbeitsplatz als Operationsschwester an einem Wunschkrankenhaus zu erhalten.

OP-JOURNAL 2000; 16: 196-202

(c) Georg Thieme Verlag Stuttgart · New York
Krankenschwestern sowie Kinderkrankenschwestern können ihren individuellen Neigungen entsprechend und unter dem Aspekt der Karriereplanung aus einer reichhaltigen Palette von Fortund Weiterbildungsmaßnahmen auswählen.

\section{Weiterbildung für Krankenpflege- personal in den Funktionsdiensten (Operationsdienst/Endoskopiedienst)}

Bereits 1979 hat die deutsche Krankenhausgesellschaft (DKG) die Empfehlung „Muster für eine landesrechtliche Ordnung der Weiterbildung und Prüfung zu Krankenschwestern, Krankenpflegern und Kinderkrankenschwestern für den Operationsdienst" verabschiedet. Im Jahre 1997 wurde eine modifizierte Fassung in Form der Empfehlung „Muster für eine landesrechtliche Ordnung der Weiterbildung und Prüfung zu Krankenschwestern, Krankenpflegern, Kinderkrankenschwestern und Kinderkrankenpflegern in den Funktionsdiensten (Operations-
dienst/Endoskopiedienst)“ erarbeitet. Diese DKG-Empfehlungen dienen als Grundlage der Anerkennung der Weiterbildungsstätten in Bundesländer, die über keine eigene landesrechtliche Regelung der Weiterbildung im Sinne dieser Empfehlungen verfügen. Die Bundesländer Niedersachsen und Hessen haben bereits eine derartige landeseigene Gesetzgebung; in Baden-Württemberg ist diese in Vorbereitung. Nach den „alten“ Richtlinien von 1979, geändert 1997 wurde bisher nur der Ausbildung von Operationspersonal Rechnung getragen. Die Richtlinien der Weiterbildung für den Funktionsdienst sehen vor, dass sowohl das Operationspersonal als auch das Endoskopiepersonal diese Fachweiterbildung gemeinsam absolvieren können. Nach gemeinsamen Ausbildungsabschnitten teilt sich die Fachweiterbildung dann in operations- und endoskopiespezifische Ausbildungsabschnitte. Selbstverständlich ist es möglich, Kurse anzubieten, in denen nur Operations- bzw. nur Endoskopiepersonal ausgebildet wird.

Ziel der Weiterbildung: Die Weiterbildung soll Krankenschwestern und Kinderkrankenschwestern mit ihren vielfältigen Aufgaben im Operationsdienst/Endoskopiedienst vertraut machen und ihnen die zur Erfüllung dieser Aufgaben erforderlichen speziellen Kenntnisse, Fertigkeiten und Verhaltensweisen vermitteln.

Teilnahmevoraussetzungen: Zugelassen werden Krankenschwestern und Kinderkrankenschwestern, die die Erlaubnis nach $\S 1$ Absatz 1 Nummer 1 oder 2 des Krankenpflegegesetzes besitzen, d.h. die über ein Kranken- oder Kinderkrankenpflegeexamen verfügen und nach der Erteilung der Erlaubnis eine in der Regel zweijährige Tätigkeit in der Krankenpflege oder Kinderkrankenpflege, davon mindestens sechs Monate im Operationsbzw. Endoskopiedienst abgeleistet haben. 
Gliederung der Weiterbildung: Die Weiterbildung findet berufsbegleitend mit theoretischem und praktischem Unterricht statt. Der Lehrgang dauert zwei Jahre und umfasst 720 Stunden theoretischen und praktischen Unterrichts sowie mindestens 2400 Stunden praktische Weiterbildung an Pflicht- und Wahleinsatzorten.

Lehrfächer und Ausbildungsinhalte: Theoretischer Unterricht: Berufswissenschaftlicher Fachbereich, Pathophysiologie, Sozialwissenschaften, angewandte Krankenhaushygiene, spezielle Pharmakologie, aktuelle Medizintechnik, übergreifende Methoden und Techniken endoskopischer Diagnostik und Therapie, Rechtswissenschaften, Krankenhausbetriebslehre und Organisationslehre, Pathophysiologie bei chirurgischen Eingriffen inkl. Erweiterung anatomischer und pathophysiologischer Kenntnisse, Methoden und Techniken chirurgischer Eingriffe.

Praktischer Unterricht: Angewandte Krankenhaushygiene, Unterricht in Instrumenten-, Geräte- und Materialkunde, Unterricht in prä-, intra- und postoperativen Maßnahmen und Verhaltensweisen, Unterricht in Verhütung von Betriebsunfällen, Erkundigungspraktika, Praxisgespräche.

Abschlussprüfung: Am Ende des Lehrgangs findet eine praktische, mündlichtheoretische und schriftlich-theoretische Prüfung statt und die Prüflinge erhalten ein Zeugnis.

Veranstalter: Jedes größere Krankenhaus bietet diese Weiterbildung in der Regel an; kleinere Kliniken oder Fachkliniken ermöglichen ihren Mitarbeiterinnen die Weiterbildung für den Operations- bzw. Endoskopiedienst, indem sie im Verbundsystem mit anderen Kliniken und im Rotationsverfahren einen Austausch der Kursteilnehmerinnen in die verschiedenen Fachgebiete sicherstellen.

Weiterbildung zur Leitung einer Funktionseinheit im Operationsdienst: Für Mitarbeiterinnen, die sich mit dem Gedanken tragen, eines Tages eine Leitungsfunktion im Operationsdienst zu übernehmen oder bereits eine solche Funktion innehaben, besteht die Möglichkeit, sich zur Leitung einer Funktionseinheit im Operationsdienst ausbilden zu lassen.

Ziel der Weiterbildung: Die Teilnehmerinnen erwerben grundlegende Kennt- nisse und Fähigkeiten, die für die Wahrnehmung von Führungs- und Leitungsfunktionen unter sich stets verändernden Rahmenbedingungen des Gesundheitswesens erforderlich sind.

Teilnahmevoraussetzungen: Die abgeschlossene Kranken- bzw. Kinderkrankenpflegeausbildung sowie eine in der Regel mindestens zweijährige Berufserfahrung im Operationsbereich.

Gliederung der Weiterbildung: Berufsbegleitend im Blocksystem oder aber als Vollzeitkurs mit mindestens 720 Unterrichtsstunden.

Lehrfächer und Ausbildungsinhalte: Pflegemanagement, Pädagogische Aspekte pflegerischen Handelns, Projektmanagement, Rhetorik und Kommunikation, Lern- und Arbeitstechniken, Mitarbeiterführung, Betriebswirtschaftslehre, Rechtskunde, Hospitationen (Quelle: DbfK, Deutscher Berufsverband für Pflegeberufe, Landsverband Baden-Württemberg e.V.).

Abschlussprüfung: Diese gliedert sich in einen schriftlichen und einen mündlichen Teil. Die erfolgreiche Teilnahme wird den Teilnehmerinnen durch ein Zeugnis bescheinigt, das den Empfehlungen der DKG entspricht.

Veranstalter: Berufsverbände, Gewerkschaften, Klinikinterne Einrichtungen der Innerbetrieblichen Fort- und Weiterbildung, andere Fort- und Weiterbildungsinstitute.

\section{Weiterbildung zur Praxisanleiterin}

Ziel der Weiterbildung: Die Weiterbildung befähigt die Teilnehmerinnen, Auszubildende der Kranken- und Altenpflege, neue Mitarbeiterinnen und Teilnehmerinnen an den Fachweiterbildungen durch pädagogische und didaktische Kompetenz gezielt anleiten bzw. einarbeiten zu können. Die Praxisanleiterin führt die punktuelle Anleitung der Schülerinnen durch und ist gleichzusetzen mit der sog. „klinischen Unterrichtsschwester".

Teilnahmevoraussetzungen: Abgeschlossene Kranken-, Kinderkranken- oder Altenpflegeausbildung und eine zweijährige Berufspraxis nach dem Examen.

Gliederung der Weiterbildung: Berufsbegleitend im Blocksystem oder aber als
Vollzeitkurs mit mindestens 430 Unterrichtsstunden.

Lehrfächer und Ausbildungsinhalte: Pädagogisch-didaktische Prinzipien, Medienkunde, Lerntheorien, Rhetorik und Kommunikation, Lernpsychologie, Entwicklungs- und Persönlichkeitspsychologie, Pflegetheoretisches Fachwissen, Ethik, Rechtskunde, Krankenhausbetriebslehre.

Abschlussprüfung: Es sind schriftliche Leistungsnachweise $\mathrm{zu}$ erbringen. Planung und Durchführung einer Anleitungs- bzw. Unterweisungssituation vor Ort. Durchführung einer Projektarbeit und deren Präsentation. Die erfolgreiche Teilnahme wird den Teilnehmerinnen durch ein Zeugnis bescheinigt.

Veranstalter: Berufsverbände, Gewerkschaften, Klinikinterne Einrichtungen der Innerbetrieblichen Fort- und Weiterbildung, andere Fort- und Weiterbildungsinstitute.

\section{Weiterbildung zur Mentorin}

Ziel der Weiterbildung: Die Mentorin ist in der Lage, die praktische Anleitung bei Auszubildenden in den Pflegeberufen sowie die Einarbeitung und Begleitung neuer Mitarbeiterinnen vorzubereiten, durchzuführen, auszuwerten, zu reflektieren und weiterzuentwickeln. Die Mentorin ist zuständig für die gezielte Schüleranleitung und die Umsetzung der praktischen Ausbildungsziele auf ihrer Station.

Teilnahmevoraussetzungen: Abgeschlossene Kranken-, Kinderkranken- oder Altenpflegeausbildung und eine zweijährige Berufspraxis nach dem Examen.

Gliederung der Weiterbildung: Berufsbegleitend im Blocksystem oder aber als Vollzeitkurs mit mindestens 120 Unterrichtsstunden (lt.Empfehlung der Baden-Württembergischen Krankenhausgesellschaft).

Lehrfächer und Ausbildungsinhalte: Berufskunde, Pädagogik/Psychologie, Gesprächsführung, Rechtskunde, Praktische Anleitung.

Abschlussprüfung/Leistungsnachweise: Es sind je ein schriftlicher, mündlicher und praktischer Leistungsnachweis zur Ausstellung des Abschlusszertifikats zu erbringen. 
Veranstalter: Berufsverbände, Gewerkschaften, klinikinterne Einrichtungen der Innerbetrieblichen Fort- und Weiterbildung, andere Fort- und Weiterbildungsinstitute.

\section{Operationstechnische Assistentin (OTA)}

Prinzipiell ist festzuhalten, dass es sich bei dieser Ausbildung primär nicht um eine Weiterbildung für Krankenpflegepersonal im Operations- bzw. Endoskopiedienst handelt. Vielmehr handelt es sich hier um eine neue Berufssparte, die ins Leben gerufen wurde, um den seit Jahren bestehenden Personalmangel in den Operationsabteilungen abzubauen. Die Deutsche Krankenhausgesellschaft(DKG) hat am 26. Juni 1996 eine Empfehlung zur Ausbildung und Prüfung von Operationstechnischen Assistentinnen und Assistenten (OTA) verabschiedet. Nach dem Willen der DKG soll diese Ausbildung zukünftig neben der bestehenden Weiterbildung für Krankenpflegepersonen im Funktionsdienst (Operations-/Endoskopiedienst) möglich sein.

Ziel der Ausbildung: Die Ausbildung soll die Schülerinnen mit den vielfältigen Aufgaben im Operationsdienst und den folgenden Funktionsbereichen: Ambulanz, Endoskopie und Zentralsterilisation vertraut machen. Die Ausbildung soll ihnen die zur Erfüllung dieser Aufgaben erforderlichen speziellen Fähigkeiten und Fertigkeiten vermitteln.

Teilnahmevoraussetzungen: Es gelten dieselben Zugangsvoraussetzungen wie für die Krankenpflege, d.h. das 17. Lebensjahr muss vollendet und die gesundheitliche Eignung zur Ausübung des Berufs muss gegeben sein.

Gliederung der Weiterbildung: Sie dauert drei Kalenderjahre, wobei es für Kranken- und Kinderkrankenschwestern mit mindestens sechsmonatiger Tätigkeit im OP eine Kürzung um 12 Monate und für Arzthelferinnen mit mindestens zwölfmonatiger Tätigkeit im OP oder operativen Bereich eine Kürzung um sechs Monate möglich ist, wenn sie zusätzlich eine Aufnahmeprüfung der OTA-Schule besteht. Insgesamt 1600 Unterrichtsstunden theoretischer und praktischer Unterricht sowie 3000 Stunden praktische Ausbildung.

Abschlussprüfung: Analog der Krankenpflegeausbildung, allerdings bestimmt der Träger der Ausbildung die/den Prüfungsvorsitzende(n) des Prüfungsausschusses.

\section{Anmerkungen}

Es handelt sich um kein anerkanntes Berufsbild. Die Anerkennung der Schulen erfolgt durch die Deutsche Krankenhausgesellschaft (DKG), solange es keine bundes- oder landesrechtlichen Regelungen gibt. Die Höhe der Ausbildungskosten entspricht der einer dreijährig-ausgebildeten Krankenpflegeperson. Von drei Jahren Ausbildungszeit verbringt die OTAAuszubildende lediglich ca. 15 Monate im Operationsdienst nach Abzug eines Pflegepraktikums, der Unterrichtszeiten, der Einsätze in der Endoskopie, Radiologie, Zentralsterilisation etc. Die OTASchulen werden zudem vorwiegend an Kliniken eingerichtet, die bereits an erheblichem Pflegepersonalmangel im Operationsdienst leiden, so dass die praktische Anleitung und Ausbildung durch das noch spärlich vorhandene Operationspersonal zusätzlich geleistet werden muss und eine weitere nicht unerhebliche Belastung des dortigen Operationspersonals darstellt.

Die Operationstechnische Assistentin (OTA) - ein neuer und vieldiskutierter Berufszweig.

\section{Warum OTA?}

Mit der Einführung des neuen Berufsbildes der OTA zur Bekämpfung des Personalmangels in den Operationsabteilungen wurde meiner Meinung nach der falsche Weg beschritten. Die Berufsverbände und die Gewerkschaft Pflege warnen vor einem „Sackgassenberuf“, weil die Absolventinnen im Falle einer Berufsunfähigkeit im Operationsdienst schwieriger oder gar nicht auf andere Arbeitsplätze im Krankenhaus versetzt werden können. Die Integration einer einjährigen Krankenpflegeausbildung in die OTAAusbildung ist für mich ebenfalls nicht überzeugend. Ich stelle mir den Fall vor, dass eine OTA aus irgendwelchen Gründen nicht mehr im Operationsdienst arbeiten kann oder will. Abgesehen vom beruflichen Prestigeverlust, dem sich vielleicht einstellenden Minderwertigkeitsgefühl und die berufliche Unterforderung in der Funktion der Krankenpflegehelferin und den daraus resultierenden persönlichen Schwierigkeiten, frage ich mich, wie eine bisher relativ gut verdienende OTA die Gehaltsdifferenz kompensieren wird? Einer Krankenschwester mit dreijähriger Ausbildung bietet sich immerhin die Alternative, im stationären Bereich oder in einem anderen Funktionsbereich eine vergleichsweise gut do- tierte Tätigkeit zu übernehmen. Des Weiteren sehe ich in der Etablierung des Berufsbildes der OTA eine über die Hintertür eingeführte Maßnahme zur Senkung der Personalkosten im Funktions- bzw. Operationsbereich, da die Bezahlung bisher unter der des Pflegepersonals im Operationsdienst liegt. An manchen Kliniken bestehen jedoch hausinterne Vereinbarungen, die die OTA gehaltsmäßig einer Krankenschwester im Operationsdienst gleichstellen. Es macht meines Erachtens überhaupt keinen Sinn, für Arbeitsplätze, die niemand mehr aus der Krankenpflege besetzen möchte bzw. für eine Tätigkeit, die offensichtlich in Anbetracht der zahlreichen Stellenausschreibungen unattraktiv ist, ein neues Berufsbild zu kreieren. Vielmehr sollten sich die Verantwortlichen vor Ort eine ehrliche Antwort auf die Frage geben „Warum lässt sich die Fluktuation des Operationspflegepersonals in unserer Operationsabteilung nicht in den Griff bekommen?" Hier gilt es, mit Sicherheit hochsensible Bereiche zu hinterfragen, $\mathrm{zu}$ analysieren und entsprechende Gegenmaßnahmen zu ergreifen, um diese Missstände auszumerzen. Folgende Gründe werden für den Ausstieg aus dem Operationsdienst sehr häufig angegeben:

die physisch anstrengende und psychisch sehr belastende Arbeit

- permanente personelle Unterbesetzung und fehlende Personalkapazitäten zum Aus- und/oder Abwechseln während lang andauernder und aufwendiger Operationen

- Fließband- und Akkordarbeit bei zunehmender Arbeitsverdichtung im Operationsdienst

- partnerschafts-, beziehungs- und familienunfreundliche Arbeits- und Dienstzeiten

- Fremdbestimmung am Arbeitsplatz (Arbeitspause, Gang zur Toilette, vorgedachte Abläufe sowie wenig oder keinen Einfluss seitens der Pflege auf die Gestaltung des OP-Programms

- Fehlen von Faktoren, die von Arbeitspsychologen als berufs- und arbeits (platz-)zufriedenheitsfördernde Bestandteile eingestuft werden wie z.B. Kreativität, Selbstorganisation, Verantwortungsübernahme, eigenständiges Denken, Miteingebundensein in Entscheidungen etc.

- reduzierte soziale Kontakte zu anderen Abteilungen bzw. Berufsgruppen

- Anonymität in großen Operationsabteilungen

- Unterbezahlung für eine hochspezialisierte Facharbeitertätigkeit (z. B. Regel- 
arbeitszeit im Nachtdienst an Großkliniken mit einer zu versteuernden Nachtdienstzulage von DM 2,50 pro Arbeitsstunde

- der Theorie-Praxis-Konflikt, d.h. die Operationsabteilung ist nicht auf „dem neuesten Stand“. Fachweitergebildetes Personal verfügt über das aktuelle Wissen, das in der Operationsabteilung aus vielerlei Gründen noch nicht in die Praxis umgesetzt wurde

- fehlende oder veraltete Standards

a fehlendes Mitarbeiter-Einarbeitungskonzept

keine Mentorinnen oder Praxisanleiterinnen

- Mitversorgung des Ambulanzbereichs in kleinen Krankenhäusern durch das Operationspersonal im Bereitschaftsdienst der Stufe A und B und Weiterarbeiten am nächsten Morgen trotz mehrmaliger Inanspruchnahme während des Bereitschaftsdienstes)

- keine Entlastung durch (zentrale) Hilfsdienste oder Versorgungsassistentinnen, wie z. B. im Stationsbereich

- hierarchische Strukturen und entsprechender Führungsstil sowie fehlende oder unzureichende Kenntnisse im Bereich OP-Management, Personalentwicklung (PE) und Organisationsentwicklung (OE) seitens der OP-Leitung

- Fortbildungsmaßnahmen beschränken sich auf Produktvorstellungen durch Firmen, d. h. qualitativ und quantitativ unzureichende Fortbildungsmaßnahmen im Bereich der Mitarbeiterführung, EDV, OP-Management, Rhetorik und Kommunikation, Konfliktmanagement, Stressmanagement, Entspannungstechniken, Pflegeforschung, etc.

problemreiche Zusammenarbeit zwischen Ärzteschaft und OP-Pflegepersonal sowie Geringschätzung des OPPflegepersonals durch die Ärzteschaft, verbale Entgleisungen seitens einiger Ärzte, weitere atmosphärische Störungen in den Operationsabteilungen durch offenes Austragen abteilungsinterner Querelen und Rivalitäten seitens der Ärzteschaft vor dem OP-Personal sowie vom Pflegepersonal ungewollte Duz-Form durch einige Ärzte

- Übernahme berufsfremder Tätigkeiten, d.h. das OP-Fachpersonal findet sich häufig in der Rolle „des billigen Mädchen für alles“ z.B. putzen des OP-Saales im Nacht- oder Bereitschaftsdienst. Haben Sie schon einmal eine Bankangestellte beobachtet, die am Ende ihres Arbeitstages ihren gläsernen Bankschalter putzt? Oder eine
Stewardess, die am Ende eines Fluges die Passagierkabine aufräumt?

• „eingefahrene“und „alteingesessene“ pflegerische OP-Teams, die den Neulingen den Einstieg in die Tätigkeit bzw. ins Team erschweren

a keine oder zu geringe Einsatzzeiten der Auszubildenden der Kranken- und Kinderkrankenpflege im Operationsdienst, so dass es zunehmend schwieriger wird, neue Mitarbeiterinnen für den Operationsdienst zu interessieren und zu gewinnen

Gezieltes Personalmanagement ist auch im Operationsdienst von größter Bedeutung.

Gezieltes Personalmanagement stellt in zunehmendem Umfang die entscheidende Einflussgröße des Erfolgs eines Unternehmens dar. Der Betrieb Krankenhaus, der sehr differenzierte Dienst- bzw. Serviceleistungen erbringt, wird zukünftig nur dann betriebswirtschaftlich erfolgreich und gegenüber anderen Kliniken konkurrenzfähig sein, wenn für die Weiterentwicklung der vorhandenen „human resources" ein qualitativ und quantitativ hoch angesiedeltes Weiterbildungsangebot besteht. Das vorhandene Mitarbeiterpotential wird oft falsch eingeschätzt. Mitarbeiterförderung geschieht nicht gezielt und so kommt es zu beträchtlichen betriebswirtschaftlich fragwürdigen Fehlinvestitionen in Form von Anwerbungs-, Gehalt-, Einarbeitungs-, Weiterbildungs- und evtl. Abfindungskosten. In den vergangenen 20 Jahren seit Bestehen der Fachweiterbildung für den Operationsdienst sind die Anforderungen an das Personal durch Hochtechnisierung des Operationsdienstes, neue Operationstechniken, den wirtschaftlichen Druck und die zunehmende Konkurrenz der Kliniken untereinander beträchtlich gestiegen und haben sich immens und rasant verändert. Die Fachweiterbildung für den Operationsdienst ist unbestritten in der vorliegenden Konzeption ein adäquates Instrument zur Heranbildung fachlich versierter und handlungskompetenter Mitarbeiterinnen für den Operationsdienst. Jedoch werden neben fachlicher Spezialqualifikation zunehmend auch sachliche und soziale Grundqualifikationen verlangt, die es ermöglichen, den komplexen Zusammenhang des konkreten Arbeitshandelns zu verstehen und $\mathrm{zu}$ beherrschen. Solchen Grundqualifikationen (= Schlüsselqualifikationen) - seit längerem im Zentrum der berufspädagogischen Diskussion sind z.B. abstraktes und symbolisches
Denkvermögen, Diagnose- und Planungsfähigkeit, Erfassen von Zusammenhängen, Flexibilität, Kooperations- und Konfliktfähigkeit. Die curriculare (auf den Lehrplan bezogen) Konzeption der Weiterbildungsrichtlinien für den Operationsdienst orientiert sich zu stark an der Vermittlung fachlicher Qualifikation; meines Erachtens wird die Schulung der Schlüsselqualifikationen vernachlässigt und ist mit der zur Verfügung stehenden Anzahl von Unterrichtsstunden nicht zu realisieren. Die berufliche Ausbildung muss zur Vermittlung personaler, formaler und sozialer Fähigkeiten auch offene und komplexe Lernsituationen bieten, in denen übertragendes Denken entwickelt wird. In entsprechenden Situationen können die Auszubildenden an komplexen Aufgaben den Lernprozess selbständig gestalten. Der Ausbilder wird Moderator des Lernprozesses. Ein Kata$\log$ möglicher Schlüsselqualifikationen (=Basiskompetenzen nach Schelten) zeigt, was darunter zu verstehen ist:

Materiale Kenntnisse und Fähigkeiten: Berufsfachliche Kenntnisse und Fertigkeiten erheblicher Breitenwirkung wie Messtechnik, Arbeitsschutz, Maschinen- und Werkzeugpflege, Zeichnungslesen, Ursachen erkennen (Fehler, Störungen etc.), ökonomisch arbeiten, Durchblick, technische Unterlagen erstellen, Planen, Steuern, Lenken, Kontrollieren, Ergebnisfeststellung, Beurteilung u.a.

Kognitive Fähigkeiten: Analytisches und synthetisches Denken, Lernfähigkeit, Kreativität, Transfer, Problemlösen, Beurteilungsfähigkeit, Kritik u.a.

Psychomotorische formale Fähigkeiten: Koordination, Reaktion, Kondition, manuelle Geschicklichkeit, Konzentration, Materialbehandlung, Stressbewältigung, Sensibilität u.a.

Personale Fähigkeiten: Zuverlässigkeit, Präzision, Gewissenhaftigkeit, Arbeitsqualität, Verantwortungsbewusstsein, Kritikfähigkeit, Flexibilität, Selbständigkeit, Selbstvertrauen, Kommunikationsfähigkeit, positives Denken u.a.

Soziale Fähigkeiten: Aufrichtigkeit, Teamfähigkeit, Kooperationsbereitschaft, Toleranz, Fairness, Konfliktfähigkeit u.a. 


\section{Neukonzeption der Weiterbildung für Krankenpflegepersonen in den Funktionsdiensten (Operationsdienst/ Endoskopiedienst)}

Neue Anforderungen im Arbeitsprozess bedingen jedoch neue Schulungsinhalte. Neben der Forderung nach fachlicher Schulung besteht - wie bereits erwähnt - verstärkt die Forderung nach Schulung der Selbst- und Sozialkompetenz der Weiterbildungsteilnehmerinnen. Unter dem Aspekt der Personalentwicklung (PE) hinsichtlich der Schulung und Förderung der persönlichen und sozialen Potentiale der einzelnen Mitarbeiterinnen, müssen die Weiterbildungsrichtlinien meiner Meinung nach eine entsprechende Nachbesserung erfahren. Diese könnte darin bestehen, die Anzahl der Unterrichtsstunden von derzeit 720 auf ca. 800 anzuheben. In den letzten Jahren gab es umwälzende Neuerungen im Bereich des Operationsdienstes, aber auch in der Krankenhausorganisation, d.h. neue Unterrichtsinhalte sind zu vermitteln, für die aber keine weiteren Unterrichtsstunden in den neuen Richtlinien vorgesehen sind. Die von mir geforderten zusätzlichen 80 Unterrichtsstunden sind inhaltlich u.a. mit folgenden Themenkomplexen zu besetzen: Pflegeforschungsprozess/-methoden, Projektmanagement, Arbeit in Arbeits-/Projektgruppen, Moderationstechniken, Präsentationstechniken und Umgang mit Präsentationsmedien, Rhetorik/Kommunikation, Konfliktmanagement, problemanalytisches Denken und problemlösungsstrategisches Handeln, Öffentlichkeitsarbeit. Stellt man die theoretischen und praktischen Lehr- und Lerninhalte gemäß den Weiterbildungsrichtlinien von 1979 und 1997 gegenüber, so ist festzustellen, dass in den „alten“ Richtlinien 20 Unterrichtsstunden „Pädagogische, soziologische und psychologische Aspekte" zur Verfügung standen; in den neuen Richtlinien sind es 30 Unterrichtsstunden als einziges Novum. Bisher bereits unterrichtete Themen erscheinen in den neuen Richtlinien von 1997 unter anderen Themenkomplexen im Lehrplan. Im Bundesland Baden-Württemberg werden die Fachweiterbildungen zukünftig im Rahmen einer eigenen landesrechtlichen Gesetzgebung geregelt. Dies ist in einigen anderen Bundesländern bereits der Fall. Dies bedeutet, dass die DKG zukünftig nicht mehr für die Fachweiterbildungsstätten in Baden-Württemberg zuständig sein wird. Betrachtet man den „Entwurf der Verordnung des Sozialministeriums über die Weiterbildung in den
Berufen der Kranken- und Kinderkrankenpflege für den Operationsdienst und Endoskopiedienst“", so stellt man fest, dass die DKG-Empfehlungen fast wortwörtlich übernommen wurden, ohne zu hinterfragen, ob diese überhaupt noch zeitgemäß sind. Die in den aktuellen Weiterbildungsrichtlinien für den Operationsdienst vorgesehenen 30 Unterrichtsstunden im Fachbereich pädagogische, soziologische und psychologische Aspekte sind niemals ausreichend, um Schlüsselqualifikationen $\mathrm{zu}$ vermitteln. Bei diesem Stundenkontingent ist es lediglich möglich, theoretische Unterrichtsinhalte vorzustellen, aber für eine nachhaltige praktische Einübung bzw. Umsetzung mit anschließender Evaluation in der Gesamtgruppe fehlt effektiv die Zeit. In diesem Zusammenhang denke ich beispielsweise an ein Kommunikationstraining, in dessen Rahmen mit den Kursteilnehmerinnen das Führen eines Kritik-, Beurteilungs- oder Verhandlungsgespräches eingeübt werden soll.

Qualitätssicherung im Bildungsmanagement des Krankenpflegepersonals im Operationsdienst als die Herausforderung der Zukunft.

In Zeiten knapper finanzieller Ressourcen und sich schnell verändernder betrieblicher und berufsspezifischer Anforderungen sollten die Pflegedienstleitungen bzw. das leitende OP-Personal als Budgetverantwortliche unbedingt daran interessiert sein, dass bei einer derart hohen finanziellen Investition in die Mitarbeiterinnen - ca. DM 35000,- pro Kursteilnehmerin - abteilungs- und/oder unternehmenszielorientiert fachweitergebildet werden. Das Leitungspersonal sollte den hohen finanziellen/personellen Aufwand für die Fachweiterbildung und den daraus resultierenden Gewinn für den Betrieb bzw. die Operationsabteilung hinterfragen. Der Rahmenlehrplan gibt zwar spezielle Themen vor, die jedoch von der Leitung der Fachweiterbildungsstätte und den jeweiligen Dozenten je nach deren Qualifikation mit entsprechenden Inhalten mehr oder weniger effektiv gefüllt werden. Der Prozess der Qualitätssicherung darf vor dem (Weiter-)Bildungsgeschehen nicht halt machen. Qualitätssicherung im Bildungsmanagement des OP-Bereichs steht für den Trend zur Orientierung an den Weiterbildungsbedürfnissen der Teilnehmerinnen und der Operationsabteilungen, d.h. es gilt die Bedürfnisse der Kunden der Weiterbildungsstätten zu erfüllen. Kundenorientierung macht es immer wieder er- forderlich, dass betriebliche Erfordernisse zur Erfüllung der Kundenzufriedenheit und die Weiterbildungs- bzw. Schulungsbedarfe der Mitarbeiterinnen zur Erlangung der spezifischen Handlungskompetenz in Einklang gebracht werden im Zusammenspiel der Weiterbildungsleitung, dem leitenden OP-Personal und der Pflegedienstleitung sowie evtl. der Ärzteschaft. Um Qualität im Fort- und Weiterbildungsprozess zu erlangen, braucht es konkrete Kriterien und Umsetzungsstrategien im Rahmen der Unterrichtsplanung und -gestaltung. Wie eingangs bereits erwähnt, regeln in Bundesländern ohne ein eigenes Landesgesetz die DKG-Richtlinien die Organisation einer Fachweiterbildungsstätte sowie die Durchführung der Weiterbildung an den Kliniken. Länder wie z. B. Niedersachsen und Hessen verfügen über ein derartiges Landesgesetz. In Baden-Württemberg existiert seit 1995 das Landespflegegesetz und das Sozialministerium arbeitet zur Zeit an einer Rechtsverordnung. Um eine schnelle Umsetzung in BadenWürttemberg zu erreichen, orientiert sich das Sozialminsterium sehr stark an den DKG-Richtlinien. Wie bereits dargestellt, dienen die Ausbildungsinhalte der DKG-Richtlinien in erster Linie der Schulung der Fachkompetenz der Teilnehmerinnen; der Schulung der zukünftig so sehr wichtigen Sozial- und Selbstkompetenz der Mitarbeiterinnen wird zu wenig Rechnung getragen, da 30 Stunden „Sozialwissenschaften“ wenig Raum zur Vermittlung des entsprechenden Wissens und der Einübung analoger Techniken vorsieht. Im Zuge der Neufassung bzw. Überarbeitung der DKG-Richtlinien im Jahre 1997 sowie bei der momentanen Ausarbeitung der Rechtsverordnung für Baden-Württemberg sind meiner Meinung nach einige Punkte, die schon immer in der Formulierung und Auslegung sehr schwammig waren, vernachlässigt worden. Diese möchte ich ganz kurz darstellen:

\section{Der Eintritt des Krankenpflegepersonals in die Fachweiterbildung für den Funktionsdienst (Operationsdienst) Endoskopie)}

Die Zulassungsbestimmungen sehen vor, dass eine ausgebildete Krankenschwester eine zweijährige Berufserfahrung nach dem Krankenpflege-Examen vorweisen muss und davon mindestens sechs Monate im Operations- bzw. Endoskopiedienst. Ausnahmen in Härtefällen sind möglich. Ich halte diese Vorschrift für inakzeptabel, da eine Krankenschwester 
sieben Ausbildungs- bzw. Berufstätigkeitsjahre bis zur Erreichung des Status der Fachschwester für den Operationsbzw. Endoskopiedienst benötigt. Eine Auszubildende zur OTA jedoch, die vor ihrer Ausbildung unter Umständen noch nie im Krankenhaus gearbeitet hat, wird sofort zur praktischen Ausbildung im Operations- bzw. Endoskopiedienst zugelassen. Es wird argumentiert, dass eine Krankenschwester auf dem Stellenplan angerechnet ist, während die Auszubildenden zur OTA ähnlich wie Auszubildende in der Krankenpflege einem anderen Anrechnungsschlüssel unterliegen. Dieses ist kein stichhaltiges Argument, da in Operationsabteilungen mit entsprechendem Personalmangel jede nur verfügbare Arbeitskraft mobilisiert und zur Bewältigung des Operationsprogramms eingeplant wird.

\section{Die Pädagogische Qualifikation der Leitung der Fachweiterbildungsstätte}

Diese ist endlich näher spezifiziert, d.h. die Leitung einer Weiterbildungsstätte muss eine Weiterbildung zum Lehrer für Pflegeberufe sowie die entsprechende Fachweiterbildung (z.B. für den Operationsdienst) nachweisen können. Leider wird diese Maßgabe dadurch zunichte gemacht, dass in Bezug auf die pädagogische Qualifikation der pflegerischen Leitung der Fachweiterbildungsstätte eine der Kompetenzen möglichst, d.h. dass z.B. ein Lehrer für Krankenpflege aus der Krankenpflegeschule als pädagogische Leitung der Fachweiterbildungsstätte pro forma eingesetzt werden kann und eine Pflegeperson mit entsprechender Fachweiterbildung die Fachkompetenz erfüllt. Bei einer derartigen Konstellation ist die pädagogisch fundierte Erwachsenen(aus-)bildung eher ein Zufallsprodukt des individuellen Engagements bzw. des individuellen Lehrertalents der nicht pädagogisch weitergebildeten Fachweiterbildungsleitung. Eine fundierte pädagogische, didaktische und fachdidaktische Qualifikation liegt nicht vor. Von Qualitätssicherung im Bildungsmanagement der Fachweiterbildung kann keine Rede sein. Aus dem jeweiligen Landesgesetz bzw. aus der Ausbildungsverordnung sowie aus den DKG-Richtlinien müsste eindeutig ersichtlich sein, dass die leitende Pflegekraft einer Fachweiterbildungsstätte sowohl für die Durchführung der administrativen/organisatorischen, currciularen und konzeptionellen Aufgaben wie auch für die Gestaltung des Unterrichtes über die entsprechende Fachweiterbildung und mindestens über eine
Ausbildung zur Lehrerin für Krankenpflege verfügen muss! Diese Forderung begründe ich damit, dass im Zuge der Qualitätssicherung im Bildungsmanagement eindeutig definiert sein muss, durch welche qualifizierende Weiter- bzw. Ausbildungsmaßnahme die pflegerische Leitung der Fachweiterbildung ihre pädagogische, didaktische bzw. fachdidaktische Kompetenz erworben hat. Meiner Meinung nach wird dem Anspruch des professionellen Bildungsmanagements im Rahmen der Weiterbildung für den Funktionsdienst dann keine Rechnung getragen, wenn die Weiterbildungsleitung nicht über die Zusatzqualifikation „Lehrer für Krankenpflege“ verfügt und als Konsequenz daraus folgende Kriterien in unzureichender Weise oder gar nicht erfüllt werden:

- Beherrschung einer Vielzahl von Unterrichtsmedien und -methoden. Als Schlagwort in diesem Zusammenhang „multimediale und multimethodischer Lehrkompetenz"

Weg vom Lehrervortrag bzw. Frontalunterricht, d. h. der Lehrer redet, die Schüler hören zu! Hin zur teilnehmerorientierten und teilnehmeraktivierenden Unterrichtsgestaltungskompetenz!

- Gezielte Ermittlung der Unterrichtsbzw. Schulungsbedarfe entsprechend der Teilnehmervoraussetzungsanalyse und deren entsprechende Umsetzung durch ein adäquates Unterrichtsarrangement

- Erfüllung der Lernzieltaxonomie, d.h. vorwiegend kognitiv und wenig pragmatisch geprägte Lernzielformulierungen müssen verstärkt affektive Elemente zugeführt bekommen. Die Unterrichtsgestaltung muss genau definierte und überprüfbare Veränderungen der Handlungsweisen, der Einstellungen und der Haltungen bei den Kursteilnehmerinnen durch entsprechende Unterrichtskonzeption und erwachsenenbildungsspezifische Unterrichtsmethodik bewirken.

- Methoden der pädagogischen Unterrichtserfolgsanalyse (Unterrichtsevaluation) und bei Bedarf die Neukonzeption des unterrichtlichen Arrangements müssen beherrscht werden.

- Der Theorie-Praxis-Konflikt muss durch geeignete Maßnahmen nach haltig abgebaut werden können.

\section{Praxisanleiterinnen/Mentorinnen}

Die Ausführungsbestimmungen der DKG sehen zum Beispiel einen Schlüssel von $1: 10$ vor, d.h. dass jede Fachweiterbildungsstätte über eine Praxisanleiterin/ Mentorin pro 10 Kursteilnehmerinnen verfügen muss. Dieser Schlüssel ist meiner Erfahrung nach zu niedrig bemessen, um allen Kursteilnehmerinnen eine fundierte praktische Begleitung bzw. Ausbildung zukommen zu lassen. Nur eine geringe Anzahl von Mitarbeiterinnen im Operationsdienst bekunden ein Interesse daran, sich zu Praxisanleiterinnen bzw. Mentorinnen weiterbilden zu lassen, da sie nicht bereit sind, neben der belastenden Arbeit im Operationsdienst, auch noch Praxisanleiterinnen/MentorinnenAufgaben zu übernehmen. Die Mehrarbeit der Mitarbeiterinnen sehen zusätzliche Belastungen wie z.B. vermehrten Stress und Ärger sowie Auseinandersetzungen mit den Fachweiterbildungsteilnehmerinnen bzw. Auszubildenden der Krankenpflege und der OTA-Ausbildung auf sich zukommen. Ganz abgesehen von der Tatsache, dass keine weitere Entlohnung für die Praxisanleiterinnen/ Mentorinnen-Tätigkeit oder eine prozentuale Freistellung von der eigentlichen Arbeit vorgesehen ist. Es gilt als vielfach belegt, dass Praxisanleiterinnen/Mentorinnen zur betrieblichen Qualitätssicherung beitragen, indem sie dafür sorgen, dass einheitliche Arbeitstechniken bzw. -methoden sowie Standards ein- und aufrechterhalten werden.

\section{Die Aufnahme der Durchführung eines Pflegeforschungsprojektes in den Lehrplan}

Eine pädagogisch sinnvolle Strategie bzw. Methode zur Vermittlung weiterer Schlüsselqualifikationen im Bereich der Selbst- und Sozialkompetenz im Rahmen einer Fachweiterbildung, stellt die Durchführung eines Pflegeforschungsprojektes dar. Die Konzeption der Fachweiterbildung für den Operationsdienst sollte es ermöglichen, den Fachweiterbildungsteilnehmerinnen den Pflegeforschungsprozess näher zu bringen. Begründung:

- der Pflegeforschungsprozess als ein Mittel der Pflegeprofessionalisierung

- die Pflegeforschung zur Verbesserung der Krankenpflege, um z.B. festzustellen, welche Pflege- und Arbeitstechniken am erfolgreichsten und effektivsten und welche Organisationsabläufe zeit-, geld- und personal-ökonomisch sind

- Horizonterweiterung durch regionale und überregionale Pflegeforschungskongresse in Deutschland und durch die Schaffung eines Forums zur Vorstellung der aktuellen Pflegeforschungsergebnisse 
die Möglichkeit der Doppelbeschäftigung der Pflegenden in Krankenpflege und Pflegeforschung

Folgende Unterrichtsinhalte sind im Rahmen der Schulung des Pflegeforschungsprozesses zu unterrichten: Problemauswahl, Benennung des Forschungsziels, Informationssuche, Literaturstudium, Hypothesenbildung, Kenntnis der Forschungsmethoden, Methoden der Datenerhebung, Datenanalyse, Ergebnis, Schlussfolgerungen, Empfehlungen, Verschriftlichung und graphische Darstellung des Forschungsberichtes mittels EDV, Ergebnisumsetzung, Präsentation und Veröffentlichung des Forschungsberichtes.

\section{Schlusswort}

In den letzten Jahren wurden die Bedingungen im Umfeld der Berufs(aus-)bildung sehr starken Veränderungen unterworfen. Die Anforderungen an die berufliche Weiterbildung steigen. Diese Anforderungen können durch die vorliegende Konzeption der Fachweiterbildung für den Operationsdienst und durch das bestehende (Weiter-)Bildungsangebot für das Operationspersonal zukünftig nicht mehr bewältigt werden. Für die in zunehmendem Maße geforderten Qualifikationen wie selbständiges Lernen, Flexibilität, vernetztes Denken und eigenverantwortliches Handeln müssen im Bereich des Operationsdienstes neue Weiterbildungskonzepte auf den Weg gebracht werden. Der ganzheitliche Einblick in den unternehmerischen Ablauf geht verloren durch fortschreitende Spezialisierung und durch den weiterhin zunehmenden Einsatz von EDV am Arbeitsplatz. Um diesen neuen Anforderungsprofilen entsprechen zu können, sind in den letz- ten Jahren vielfältige Schlüsselqualifikationskonzepte entwickelt worden, die den Arbeitnehmerinnen helfen sollen, schnell auf unvorhersehbare Veränderungen im beruflichen Umfeld reagieren zu können. Im Bildungsmanagement ist Handlungsorientierung gefragt, d.h. eine bestimmte Art der Gewinnung und Festlegung von Lernzielen und -inhalten, sowie/oder eine bestimmte methodische Gestaltung der Lernsituation. Die Fachweiterbildungsteilnehmerinnen sollen im handlungsorientierten Unterricht ihre Lernprozesse selbständig, teilweise selbstorganisiert und interaktiv mit ihrer Umwelt gestalten. Zur Vermittlung von Schlüsselqualifikationen ist eine ganzheitliche Vorgangs- und Ablaufsverarbeitung im Unterricht notwendig. Berufliches Lernen soll verstärkt als handlungsorientierter Prozess in komplexen, ganzheitlichen Lernumgebungen organisiert werden. Die Lernenden müssen sich in der Lernumgebung selbsttätig und selbständig mit den Lerninhalten auseinandersetzen, weil selbst Erlerntes und selbst Erfahrenes besser behalten wird als das im Frontalunterricht vorgetragene Faktenwissen. Handlungsorientierung in der beruflichen Bildung beinhaltet die Wiederherstellung der Einheit von Theorie und Praxis, Lernen anhand komplexer, ganzheitlicher Aufgabenstellungen sowie die Persönlichkeitsbildung der Lernenden. Für die Fachweiterbildungen bedeutet eine zunehmende Handlungsorientierung, dass sie Lehr- und Lernarrangements entwickeln muss, die authentische, simulierte und/oder symbolisch repräsentierte Arbeitshandlungen ermöglichen. Die Schlüsselqualifikationen müssen durch fächerübergreifende und transferfördernde Lernprozesse gesichert werden. Die berufsübergreifenden allgemeinen Kompetenzen müssen erwei- tert werden. Der handlungsorientierte Unterricht setzt eine veränderte Lehrerrolle voraus. Der frontal unterrichtende Lehrer schlüpft in die Rolle des Lernberaters.

\section{Literatur}

1 Lobiondo-Wood G, Haber J. Pflegeforschung: Methoden, kritische Einschätzung und Anwendung Berlin, Wiesbaden 1996

2 Mönig W. Bundesvorsitzender der Gewerkschaft Pflege. Neuer Ausbildungsberuf im Krankenhaus: Der Operationstechnische Assistent. 1996

3 Paulik H. Schlüsselqualifikationen/Basiskompetenzen nach Schelten aus „Der Ausbilder im Unternehmen“ - Berufsbild, Prüfungsvorbereitung, pädagogisches Grundwissen. 12. überarbeitete Auflage 1991

4 Reetz L. Zum Konzept der Schlüsselqualifikationen in der Berufsausbildung, Begründung und Legitimation eines pädagogischen Konzeptes. In: Berufsbildung und Wissenschaft und Praxis. 1989

5 Sozialministerium Stuttgart. Verordnung des Sozialministeriums über die Weiterbildung in den Berufen der Kranken- und Kinderkrankenpflege für den Operationsdienst und Endoskopiedienst. Stuttgart 17. Januar 2000

6 Staudt E. Defizitanalyse betrieblicher Weiterbildung. In: Schlafke W, Weiß R. (Hrsg.) Tendenzen betrieblicher Weiterbildung. Köln, 1990

7 Zahorka H.-J. Libertas Paper, Berufliche Weiterbildung und Wettbewerbsfähigkeit: Entwicklungen, Tendenzen, Möglichkeiten. Sindelfingen 1994

8 Zintl V. Lernen mit System. München 1998

\section{R. Huttelmaier}

Fachpfleger für den Operationsdienst Lehrer für Krankenpflege

Leiter der Fachweiterbildung für den Operationsdienst

Universitätsklinikum Tübingen

Scheefstr. 19

72076 Tübingen 